were other, perhaps more appropriate, responses to the problem of Down's syndrome. Since this would call into question any programme directed at identification and termination of affected pregnancies, it would be logical to resolve this dilemma before any programme was started.

The authors thank members of staff of the Glasgow and West of Scotland Genetic Advisory Centre for providing detailed information about their work, Mr R G Milne for several original ideas, Dr F A Boddy, Professor M A Ferguson-Smith, and Professor G T Stewart for giving helpful advice, and Mrs F Sinclair for secretarial help.

\section{References}

${ }^{1}$ Drillien, C M, Jameson, S, and Wilkinson, E M, Archives of Disease in Childhood, 1966, 41, 528

2 Mental Handicap, 17 . London, Office of Health Economics, 1973.

3 Economic Progress Report. London, Information Division of Treasury, 1974

4 Lindsjö, A, Acta Paediatrica Scandinavica, 1974, 63, 571.

5 General Register Office, Scotland, Report of Registrar General for Scotland, Edinburgh, HMSO, 1973.
5 General Register Office, Scotland, Report of Registrar General for Scotland, Edinburgh, HMSO, 1973.

7 Penrose, L S, and Smith, G F, Down's Anomaly, p 152. London, Churchill, 1966.

${ }^{8}$ Collmann, R D, and Stoller, A, Fournal of Mental Deficiency Research, $1963,7,53,60$.

${ }^{9}$ Fabia, J, and Drolette, M, Fournal of Mental Deficiency Research, 1970, $14,235$.

${ }_{10}$ Ross, H S, Innes, G, and Kidd, C, Scottish Medical fournal, 1967, 12, 260

11 Carter, F A, and Hagard, S, Calculating the Costs of Down's Syndrome. University of Strathclyde Health Services Operational Research Unit. Mimeograph, 1975

12 Milne, R G, Institutional Provision for Mongolism and Spina Bifida in Scotland, submitted for publication.

13 McKeown, T, and Lowe, C R, An Introduction to Social Medicine, p 317. 2nd ed. Oxford, Blackwell, 1974.

14 Scottish Education Department, Degrees of Mental Handicap. Edinburgh, HMSO, 1961.

15 Central Statistical Office, Social Trends, London, HMSO, 1973.

16 Central Statistical Office, Annual Abstract of Statistics. London, HMSO, 1973.

17 Hagard, S, Carter, F A, and Milne, R G, British fournal of Preventive and Social Medicine, in press.

18 Ferguson-Smith, M A, et al, Health Bulletin, in press.

19 Stein, Z, Susser, M, and Guterman, A V, Lancet, 1973, 1, 305.

\title{
Problems of Childhood
}

\section{Wheezing children}

\author{
J K SARSFIELD
}

British Medical fournal, 1976, 1, 756-759

The wheezing child is a common clinical problem. Asthma is by far the commonest cause of wheezing, but other conditions must be considered, especially in the young child. These include respiratory tract infection, expecially viral bronchiolitis, inhalation of a foreign body, cystic fibrosis, primary tuberculosis, and congenital anomalies. Clinical and radiological examination will usually identify these conditions.

The role of infection in wheezing in the young child is not clear. Acute bronchitis with airways obstruction may produce a wheeze with associated respiratory distress, fever, leucocytosis, and general malaise. If these attacks recur terms such as "wheezy bronchitis," "asthmatic bronchitis," and "pseudoasthma" are applied. Antibiotics are often prescribed but with doubtful benefit. Viral infection may be responsible for some attacks, but several studies have shown that viruses can be isolated from only about a third of patients and their role in the pathogenesis is uncertain. Williams and $\mathrm{McNicol}^{1}$ made a valuable contribution to our understanding of this group of young wheezing children who have apparent preceding infection. After a prospective long-term epidemiological study they concluded that they could not separate recurrent "wheezy bronchitis" from asthma. Their evidence suggests that both conditions exhibit a common basic asthmatic disorder, but the

Department of Paediatrics and Child Health, University of Leeds, Leeds LS14 3ET

J K SARSFIELD, MD, MRCP, lecturer in paediatrics spectrum of severity varies greatly from a few mild early attacks that abate to established severe asthma persisting into adult life.

\section{Asthma}

In the absence of any widely accepted definition of asthma it seems reasonable to consider it, simply, as a constitutional disorder characterised by hyper-reactivity of the airways. Various factors may provoke this reactive state and lead to paroxysmal attacks of airways obstruction producing respiratory distress and wheezing. The basic constitutional disorder is probably biochemical and almost certainly has a genetic basis, but the precise mode of inheritance is unknown. This familial tendency, however, may help the doctor towards an earlier diagnosis and hence more appropriate management.

An understanding of the known basic mechanisms concerned in the cause of asthma is essential to proper assessment and management.

\section{Allergy}

Most asthmatic children have demonstrable allergies. The incidence of hay fever, eczema, and urticaria is much higher in these children and their families than in non-asthmatics. The fact that an acute attack of asthma may be a manifestation of an immunological hypersensitivity reaction to an external provoking antigen (allergen) has been recognised for many years. Recent advances have established that antibodies belonging to a newly recognised immunoglobulin class, IgE, are concerned. These antibodies are firmly attached to mast cells in the bronchial mucosa, and on exposure to an offending allergen the antibodyantigen reaction causes the release of vasoactive amines from 
these cells. The effect of these amines, which include histamine, is to produce bronchospasm, mucosal swelling, and excessive secretion that narrow the airways and produce an asthmatic attack.

Children may acquire sensitivities to various allergens from the earliest months of life. Food allergy is probably important in early childhood; cows' milk and eggs are commonly incriminated. The mother with a strong family history of allergic disease should be encouraged to breast-feed her baby, who will benefit by avoiding potentially allergenic cows' milk proteins and allergy to other substances will be delayed by the protective antibodies present in the mother's milk. House-dust mite, grass pollens, and domestic pets are other common potential allergens. The particular allergen(s) to which the child becomes sensitive is unpredictable, provided, of course, that there are opportunities for exposure.

It is essential that a thorough search is made to identify the offending allergens which cause symptoms in an allergic asthmatic child. The history may give valuable clues: grass pollen causes seasonal attacks; non-seasonal and especially nocturnal asthma is often due to house-dust mite allergy; and, although pet allergy may be apparent, this is not always so. Skin testing by the epidermal prick method remains the mainstay of diagnosis. This may be done by the doctor simply pricking the skin, once, through a drop of commercially available (Bencard or Dome) allergen extract solution. For inhaled allergens a negative skin test result usually excludes clinical sensitivity. A positive result with a weal and flare reaction may indicate bronchial sensitivity. In general a large skin test reaction, especially a weal diameter greater than $5 \mathrm{~mm}$, will suggest that this specific allergen is causing symptoms.

In all but the mildest cases referral to hospital is indicated for an expert assessment of the allergic state. Nasal and bronchial challenge testing allow more accurate identification of offending allergens, but they require expertise in their use and interpretation. Among recent advances, a radioimmunoassay technique of detecting specific IgE antibodies in a serum sample promises to be a reliable aid in identifying the particular allergens concerned in allergic subjects and has particular advantages in investigating the young child.

\section{Emotion}

Almost every asthmatic child will have an emotional component in the cause of his wheezing. This is usually only of minor importance and disappears with the successful management of other factors. Laughing, crying, frustration, excitement, or unhappiness, however, may provoke attacks. A few children learn how to influence their parents' attitudes by manipulating their symptoms. Only a few have gross psychiatric disturbances requiring expert advice.

\section{Infection}

Infection appears to be a common precipitating factor in the young child but may provoke wheezing at any age. It is often overdiagnosed and the preceding manifestations of allergic rhinitis-sneezing, nasal stuffiness, and discharge-are often attributed to a "common cold." Repeated bouts of proved respiratory infection should prompt a suspicion of an immune deficiency state or cystic fibrosis.

\section{Exercise}

Many asthmatic children (both toddlers and older children) wheeze after exercise. If severe, it may be a great handicap to the child's normal eagerness to participate in play and sports. Exercise to exhaustion should certainly be avoided but excess restrictions on school activities may interact, in a deleterious way, with the emotional component of the disease. The under- lying mechanisms of exercise-induced wheezing are incompletely understood, but the final common pathway is probably the mast cell and it will respond to appropirate drug treatment.

\section{Physical factors}

Extremes of environmental temperature, fog, noxious fumes, and smoke may all provoke wheezing and should be avoided. These reactions are a reflection on the hyper-reactivity of the airways of the asthmatic child.

\section{Clinical assessment}

History-A carefully taken history is essential and will show the frequency and severity of attacks and the degree of the child's handicap. Valuable information may be gained about precipitating factors, especially provoking allergens-for example, seasonal exacerbations in pollen asthma, nocturnal wheezing with mite sensitivity, or a clear-cut reaction to pet animals. Adverse factors in the child's domestic and school environment should be carefully assessed.

Examination-Special attention should be paid to a poor physique and chest deformities such as pigeon or barrel chests. These suggest severe chronic asthma and a failure of previous treatment and will usually indicate the need for expert assessment. Commonly associated conditions such as allergic rhinitis and eczema may be identified. Repeated estimations of the peak flow rates using a Wright's peak flow meter are a simple and objective guide to the severity and may be used to assess the response to treatment.

\section{Management}

Asthma in childhood usually persists for many years, but as puberty approaches there is a definite tendency for the child to "outgrow" his symptoms. Since less than $20 \%$ will continue wheezing into adult life an optimistic attitude is justified and this will often go far to relieve parental tensions. As in other chronic diseases in childhood, continuity of medical care is most desirable. The sympathetic doctor who devotes time to listen to the parents' questions and anxieties will gain their confidence. Too often parents complain of their child's exposure to numerous different doctors and drugs while at the same time they feel ill-informed about the basic nature of the child's illness. It is little wonder that despair and resignation soon set in and they become hospital "non-attenders" or send to the surgery for a bottle of "the usual" praying for the day when he will outgrow his asthma.

\section{ACUTE ATTACK}

Acute attacks of asthma vary greatly in severity and duration. The parents may do much in the early stages, especially by showing confident and understanding attitudes and never displaying any panic or expressing excess anxiety. A supply of an oral bronchodilator drug, such as salbutamol (Ventolin) or terbutaline (Bricanyl), should be kept at home and given at this stage and repeated every six hours. Aminophylline suppositories should probably not be kept at home; the absorption of the drug by this route is irregular and unpredicatble and there is a risk of overdose. When called to see a wheezing child, the general practitioner must first assess the severity of the attack and establish which drugs have been given to the child. The commonest error is to underestimate the severity of the attack. Signs of severity are tachycardia, hypoxic restlessness, and the use of accessory muscles of respiration with poor air entry. The prolonged severe episode leads to exhaustion, disorientation, and cyanosis. These are of grave importance and need immediate admission to hospital. 
After the initial assessment the decision may be made in the less severe cases to treat at home with bronchodilators. The older child will respond to intravenous aminophylline diluted and given slowly (over at least ten minutes) in a dose of 3-5 $\mathrm{mg} / \mathrm{kg}$, provided this drug has not been given in the previous eight hours, by mouth or rectally. Overdosage or rapid administration of aminophylline is dangerous: it will probably be superceded for domiciliary use by injecting either terbutaline (up to $0.25 \mathrm{mg}$ subcutaneously) or salbutamol, which is currently under trial in paediatric practice. Adrenaline has definite adverse cardiac effects and can no longer be recommended. Any failure to respond to these measures, or if the attack is prolonged with signs of exhaustion, would be indications for admission to hospital. It is wise, in these severe cases, to give hydrocortisone $100 \mathrm{mg}$ intravenously and prednisolone $20 \mathrm{mg}$ by mouth before transfer, as these take one to two hours to take effect. A full record of the drugs given, with times and amounts, should accompany the patient.

Admission to hospital allows more intensive investigations, which include $x$-ray films of the chest, estimation of blood gases, and $\mathrm{pH}$ measurements. Intensive care may be necessary and special attention paid to maintaining hydration and electrolyte balance by intravenous treatment and the correction of any acidosis with sodium bicarbonate. A moist oxygen-air mixture is given to maintain the arterial oxygen tension $\left(\mathrm{PaO}_{2}\right)$ near $10.7 \mathrm{kPa}(80 \mathrm{~mm} \mathrm{Hg})$. Drug treatment is continued. Hydrocortisone given $100 \mathrm{mg}$ intravenously every four hours, and salbutamol by inhalation in a fine mist over 10 minutes and repeated four-hourly is often helpful. Any suspected infection is treated with an appropriate broad-spectrum antibiotic. Progression into severe respiratory failure is indicated by increasing exhaustion, a depressed level of consciousness, cyanosis, and diminished breath sounds. The arterial $\mathrm{PaO}_{2}$ falls and the $\mathrm{PaCO}_{2}$ rises to levels above $8.0 \mathrm{kPa}(60 \mathrm{~mm} \mathrm{Hg})$. Ventilatory support is then necessary and for this the skills of an anaesthetist are required. Intensive treatment and monitoring are continued. The ventilator will be necessary until the airways obstruction lessens and air entry is improved.

\section{Long-term management}

The aims in long-term management are to reduce the frequency and severity of acute attacks and if possible prevent them recurring. Much can be achieved by proper long-term management.

\section{AVOIDANCE MEASURES}

The avoidance of known precipitating factors, if possible, is a common-sense, cheap, and often very effective way of reducing symptoms. Some allergens, such as grass pollen, are impossible to avoid. Others are completely avoidable and include domestic animals. In younger children the elimination of suspected offending foods from the diet may be worth a trial but this must be continued for at least a month. The success of any avoidance programme will depend on the accurate identification of the offending allergens. The commonest allergen incriminated in childhood asthma is the house-dust mite, which thrives in bedrooms and bedding. Measures aimed at reducing exposure to this organism (and its equally allergenic exoskeletons and secretions) should be advised in all cases of mite allergy. An "impervious," thick plastic, box-type, mattress cover* should be used to enclose the mite-dust within the mattress and this will also reduce the levels of active infestation. Bedding made of synthetic materials and the use of foam pillows should be recommended. The bedroom requires regular vacuum cleaning and lino or vinyl floor covering is preferred. Application of these simple measures will often end the asthmatic attacks in mitesensitive children. The removal of a family pet may be indicated *Obtainable from R J Morpeth Ltd, 39 Clayton Street, Newcastle upon Tyne NE1 5PN but this often causes emotional upset and family strife and should be advised only when there is definite evidence of its role as an allergen. A trial period of lodging it away from the home for several months is often worthwhile. Parents of children with allergic asthma should be advised not to introduce any new furry pets into the home.

\section{PROPHYLACTIC DRUG TREATMENT}

Prophylactic drug treatment may be needed if avoidance measures are impossible or have failed to control symptoms. The asthmatic child with only an occasional attack is best treated with a bronchodilator drug, such as salbutamol, $2 \mathrm{mg}$, taken as soon as symptoms appear. The parents should keep a supply of an appropriate bronchodilator drug but it should not be used as a prophylactic agent.

Sodium cromoglycate (Intal) is a particularly safe and useful prophylactic drug. It is indicated for those children with chronic asthma who often have multiple unavoidable allergies and is particularly effective in those showing labile airways obstruction. Asthma limited to the grass pollen season will usually be relieved by its use during this period. A capsule inhaled one hour before a period of exercise will often ameliorate the symptoms of post-exercise bronchospasm. The powdered contents of a capsule are inhaled using a special Spinhaler unit. The starting dose is one capsule four times daily; this may be reduced to three or two capsules daily if there is a favourable response. On the other hand, an increase to six or even eight capsules daily may be tried before abandoning the drug if the initial response is poor. Side effects are rare and mild with local irritation the most common. It is particularly important to instruct the child and his parents in the mechanics of administration and to stress repeatedly the prophylactic nature of this treatment.

Corticosteroids by aerosol-Two corticosteroid drugs, beclomethasone dipropionate (Becotide) and betamethasone valerate (Bextasol), which have high topical activities, are available as aerosol preparations for the prophylactic treatment of the child with severe chronic asthma. Their use should be restricted to those children who have had expert assessment and have failed to respond to sodium cromoglycate and to those who require continued oral steroid treatment to control their symptoms.

A reduction in the dose of regular oral steroids or even a complete weaning off may be achieved by using an aerosol steroid. The initial dose of either drug is two puffs three times daily which may be reduced or increased up to eight puffs a day, depending on the clinical response. A further temporary increase, up to 16 puffs daily, may be given if there are signs of an impending attack. An established attack will not be helped by using the aerosol and the use of oral corticosteroids will usually be necessary. Growth retardation and adrenal suppression have not been observed using recommended doses of the aerosol steroid and the possible side effect of local monilial infection is rare in children.

Systemic corticosteroids-Resort to regular treatment with an oral corticosteroid, such as prednisolone, may be occasionally necessary in the most severe cases, when the child has not responded to other drugs or is too young to co-operate with the administration of inhaled drugs. Oral corticosteroids will certainly control symptoms but the daily dose needed will almost always produce adrenal suppression and suppress growth. These can be avoided largely by giving the total two-day dose on alternate days and this is currently recommended, but there is usually some loss of clinical response. Injections of ACTH also reduce growth suppression, but injections are especially unpopular with children. The dose of oral corticosteroids must be kept to a minimum and repeated attempts must be made at dose reduction and to wean the child off this form of treatment. Any acute attack will demand a short-term increase in doses and the child should always carry some form of identification that he is on long-term steroid treatment. 


\section{HYPOSENSITISATION}

Hyporsensitisation, which requires multiple injections, has been widely used for many years but its effectiveness is still controversial. Some benefit may be obtained against grass pollen allergy, but no convincing evidence is available that it is effective against house-dust mite or food allergies. The use of multi-allergen "cocktails" cannot be recommended. Until more basic knowledge of the immunological processes involved in hyposensitisation is available and properly designed clinical trials have proved its effectiveness this method of treatment cannot be recommended.

\section{PHYSIOTHERAPY}

Instruction and practice in the correct methods of breathing and relaxation during an acute attack are necessary for children who are incompletely controlled. Undue emphasis, however, must not be placed on this aspect of symptomatic treatment. All too often asthmatic children attend local physiotherapy departments with an inevitable loss of schooling and have yet been denied basic aetiological assessment. Over-zealous "slapping and tipping" during an acute attack must be avoided.

\section{RESIDENTIAL SCHOOLING}

The need for placing asthmatic children into residential schools has decreased as a result of a better understanding of the disease and the recent advances in treatment. The child with intractable asthma will often improve after such a placement. The reasons for this will vary but may include removal from domestic allergens or psychosomatic causes, regular supervised medication, and the general improvement in the social environment.

The management of the wheezing child requires careful investigation and the proper use of available therapeutic measures. Generalised observations about treatment are useful but their application must be tempered by the individual's response. There are no "short cuts," but there are few other chronic conditions of childhood which will appropriately reward the doctor's enthusiasm.

\section{Reference}

1 Williams, H, and McNicol, K N, British Medical fournal, 1969, 4, 321.

\title{
Hospital Topics
}

\section{Incidence of tuberculosis, hepatitis, brucellosis, and shigellosis in British medical laboratory workers}

\author{
J M HARRINGTON, H S SHANNON
}

British Medical fournal, 1976, 1, 759-762

\section{Summary}

A retrospective postal survey of 21000 medical laboratory workers in England and Wales showed 18 new cases of pulmonary tuberculosis in 1971, a five-times increased risk of acquiring the disease compared with the general population. Technicians were at greatest risk, especially if they worked in morbid anatomy departments. Of the 35 cases of hepatitis, the technicians were again the occupational group most likely to acquire the disease. Microbiology staff were twice as likely to report shigellosis as those in other pathology divisions but only one case of brucellosis was reported in the whole laboratory population. A similar survey carried out in 1973 of 3000 Scottish medical laboratory workers corroborates the results from England and Wales. Medical laboratory workers continue to experience a considerable risk of

TUC Centenary Institute of Occupational Health, London School of Hygiene and Tropical Medicine, London WC1E 7HT

J M HARRINGTON, MB, MRCP, lecturer in occupational medicine (present address: medical epidemiologist, Environmental Hazards Activity, Cancer and Birth Defects Division, Bureau of Epidemiology, Center for Disease Control, Atlanta, Georgia 30333 USA)

H S SHANNON, MSC, FSS, statistician

developing an occupationally acquired infection. Improvements in staff safety and health care seem to be necessary.

\section{Introduction}

Medical laboratory work has always been a source of occupational hazards. ${ }^{1-4}$ Fires, explosions, gassings, and physical injury $^{5}{ }^{6}$ may occur in all types of laboratories, but medical establishments carry the additional risk of occupationally acquired infections. Despite the large volume of pathogenic material handled by routine diagnostic laboratories, the risk may be greatest in research laboratories, as the dangerous nature of the substances or processes may be unknown. Laboratory workers not infrequently become the first unwitting human case of such an infection. Examples include Herpes B virus,? American $Q$ fever, ${ }^{8}$ Louping ill, and Newcastle disease. ${ }^{\circ}$ Marburg disease was first described in laboratory workers ${ }^{10}$ and parenteral spread of hepatitis virus was probably first noted after an accidental inoculation with jaundiced serum in 1929.11

Few surveys of laboratory-acquired infection have been undertaken; most reports are of small outbreaks in specific laboratories. Sulkin and Pike's study of 5000 American laboratories ${ }^{1}$ suggested that brucellosis, tuberculosis, hepatitis, and enteric diseases are among the commonest laboratory-acquired infections, and these findings have been supported by other reports. ${ }^{4}$ In 1957 Reid $^{12}$ noted that British medical laboratory workers had a risk two to nine times that of the general population of 